\title{
Sentiment Analysis of Hotel Review
}

\author{
Dhore Akshada Sharad ${ }^{1}$, Dixit Shraddha Ashok ${ }^{2}$, Dixit Pratik Dattatray ${ }^{3}$, Madke Prajakta Bhagwat ${ }^{4}$ \\ BE Student, Information Technology, VPKBIET, Baramati, India ${ }^{1,2,3,4}$
}

\begin{abstract}
Now a days, social media is hot topic on research. Millions of the peoples express their views on social media. This huge data will beneficial for better product marketing. But, because of massive of volume of reviews, endusers can't read all reviews in order to solve this problem lot of researchers has been carried out sentiment analysis.

Sentiment analysis is the automated process of understanding and opinion about a given subject from return or written language. Most of sentiment analysis \& opinion mining work focuses on binary classification \& ternary classification of texts. But our novel idea is to classify the text or sentences into multiple classes. Using Hotel Reviews datasets we classify the sentences into multiple classes like happy, sad, hungry, love etc. In dataset various sentences contains the hashtags, URL's, operators it cannot change the analysis of the sentences or meaning of that sentence but it create the confusion while determining the result. So we can apply the pre-processing method to remove all these things. After that the feature extraction method is apply on the processed dataset to extract their aspects. Later, this aspects are used to calculate the positive and negative polarity in sentence. Then the model is trained on training dataset using supervised learning method. The training consist of the pairs of input and the corresponding answer vector and the current model is run with the training dataset and produces a result. By using machine learning algorithm or NLP algorithms, the classification will give the better accuracy \& these analyses will be helpful for product developer and end-user.
\end{abstract}

Keywords: Sentiment Analysis, Machine Learning, Feature Extraction

\section{INTRODUCTION}

The day by day improvement in the field of web technology people's opinion mining and perception is one of the important part of growing advancement. People express their views, ideas, and comments about product on social media i.e. Facebook, twitter. To stay exist in these computational world study of end users opinion is a supreme aspect. Sentiment analysis is one of the twitter domain for mining the user's opinion. The process of identifying and detecting subjective information using machine learning algorithm, text analysis and computational linguistics is referred as sentiment analysis. In short, the aim of sentiment analysis is extract information on the attitude of writer or speaker towards specific topic or polarity of documents. In today's era, everything is online no one is depend on other for their own requirements. But sometimes the user gets any difficulty in selection of products at that time users must need to check the reviews or comments as well as ratings of the product and select better product.

Sentiment analysis is done by three levels

1. Document level: Analysis is depends on whole documents and then express weather document is positive or negative sentiments.

2. Sentence level: It is find the sentiment polarity from short sentence.

3. Entity/Aspect level sentiment analysis performance: Ex-Rahul says my listening power is strong but $\mathrm{i}$ am getting tired very quickly.

Up til now, sentiment classification has done in binary and ternary classification in which text is classify into two classes i.e. positive and negative classes. The binary classification is not applicable for classifying polarity words it also impact on accuracy of results. So the researchers look forward for the ternary classification. With the growing of word they are not satisfy with the binary or ternary classification so they turns toward invention of multiclass. The methods which are in these paper for multiclass classification will better impact on accuracy and opinion mining.

\section{LITERATURE SURVEY}

Our day-to-day life has always influenced by what other people think. Ideas and opinions of others are always affected our own opinions. Sentiment analysis is the computational treatment of opinions, sentiments and text. On the basis of sentiment analysis, different researchers have represent the different techniques. Some are based on the machine leaning, probabilistic and some are the mixture of these two techniques. Some of them are explained in the following.

W.Gao et al. [1] he proposed the quantification method over the years. There are two main classes that is aggregative and non-aggregative methods. So, the former require the classification of each individual item as an intermediate step. Most of the methods are fall into the former class while latter are few representatives. In aggregative method, the 
Vol. 7, Issue 11, November 2018

distinction can made between the methods that use general-purpose algorithm.

Pang et al.[4] by using machine learning algorithm he represent the pioneer work to classify the text based on their sentiment polarity. In their work he can use the unigram model, bigram model and different activities in different ways to the movie reviews into the positive or negative. On the basis of positive reviews he can easily understand which movie is going to hit and which give a less profit.

Bollen et al. [5] they analyze the tweets between August 1 and December 20, 2008. They check how the social, political, cultural and economic sphere produces an effect on public mood expressed on Twitter. But the difference is that, they analyze the tweets from twitter but do not make distinction between the sources of tweets.

Pablo et. al. [6] presented variations of Naïve Bayes classifiers for detecting polarity of English tweets. There are two different variants of Naïve Bayes classifiers were built namely Baseline (trained to classify tweets as positive, negative and neutral), and Binary (makes use of polarity lexicon and classifies as positive and negative. Neutral tweets will be neglected).The features considered by classifiers were Lemmas (nouns, verbs, adjectives and adverbs), Polarity Lexicons, and Multiword from different source and Valence Shifters.

\section{SYSTEM ARCHITECTURE}

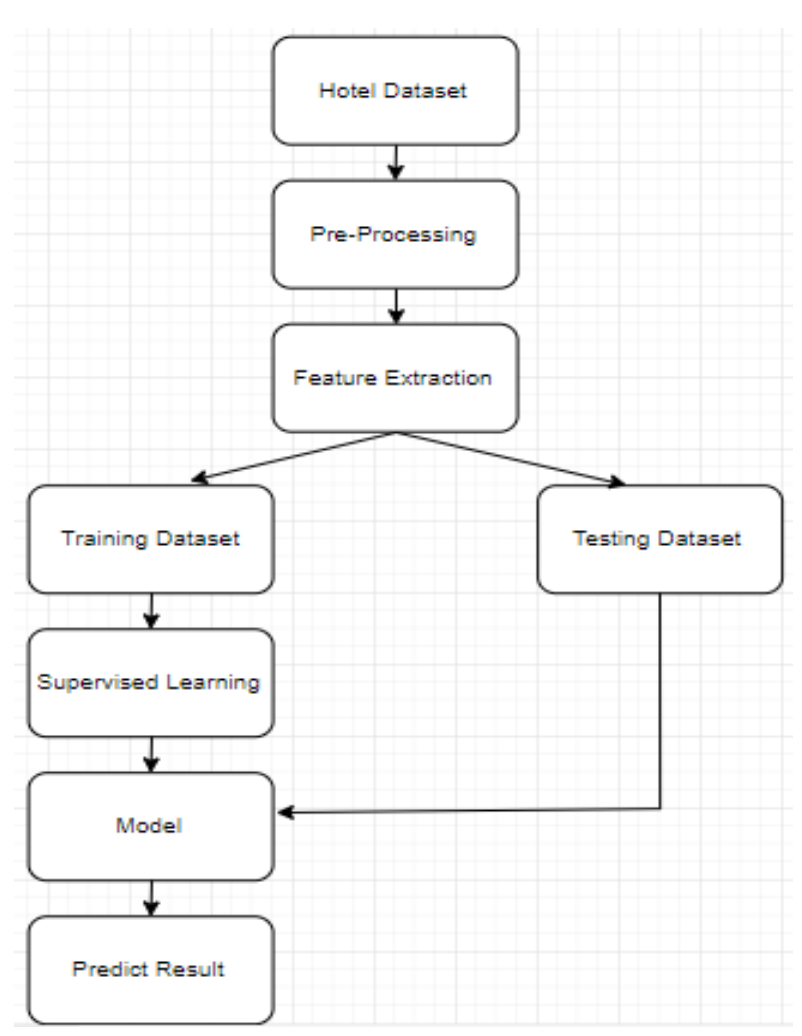

Pre-Processing: Pre-Processing is one the most important task in sentiment analysis. It will clean the dataset by reducing its complexity to prepare the data for classification. Firstly , the dataset was tokenize to split the words into tokens, then stemming will reduce the tokens into single type, for example, the word "hotels" will reduce to "hotel". The stemming process reduces redundant words in a document. In pre-processing, three tier of cleaning strategies was introduced, which is tier 1 , tier 2 and tier 3.

Tier 1: Remove stop words: At this tier, remove the articles like a, an and the since they do not play any role in determining the sentiment.

Tier 2: Remove stop words+ meaningless words: Meaningless words means that type of words that doesn't give any effect on analysis but they create confusion during conversion of text file into numeric file. From experiment, it is observed that the data contains more than hundred meaningless words such as special characters (@, \#), date $(08 / 10 / 18)$, and no meaning words $(a+, a-, b+)$.

Tier 3: Remove stop words+ meaningless words+numbers+words less than 3 character: At this stage, more cleaning strategies were performed. Besides removing the stop words and meaningless words, numbers and words that the length is less than three characters was also removed. 
Vol. 7, Issue 11, November 2018

Feature Extraction: Pre-processed dataset has many different properties so it is hard to understand. In feature extraction method, we extract the aspects from processed dataset. Later this aspects are used to calculate the positive and negative polarity in a sentence which is used to determine opinion of individuals using model like unigram and bigram. For representing the key feature the machine learning technique is require for processing of text or document. These key feature are considered as feature vector which are used for classification of text. Some examples are:

1. Parts of Speech Tags- Part of speech like objectives, adverbs and some groups of nouns and verbs are good indicators of subjectivity and sentiment. We can generate dependency pattern by parsing or dependency trees.

2. Opinion words and Phrases- Some phrases and idioms, apart from specific words which convey sentiments can be used as features.

3. Position of Terms- The position of term which is in text can affect on how much the term makes difference in overall sentiment of text.

4. Negation- Negation is important but difficult feature to interpret presence of negation usually changes the polarity of the opinion. e.g.-I am not happy.

\section{NAÏVE BAYES THEOREM}

Bayes theorem provides a way for calculating posterior probability $\mathrm{P}(\mathrm{c} \mid \mathrm{x})$ from $\mathrm{P}(\mathrm{c}), \mathrm{P}(\mathrm{x})$ and $\mathrm{P}(\mathrm{x} \mid \mathrm{c})$.

$$
\mathbf{P}(\mathbf{c} \mid \mathbf{x})=\frac{\mathbf{P}(\mathbf{x} \mid \mathbf{c}) \mathbf{P}(\mathbf{c})}{\mathbf{P}(\mathbf{x})}
$$

Assumption is made that the events are conditionally independent.

Here, $\mathrm{P}(\mathrm{c} \mid \mathrm{x})$ is posterior probability.

$\mathrm{P}(\mathrm{c})$ is the prior probability class.

$\mathrm{P}(\mathrm{x} \mid \mathrm{c})$ is likelihood.

$\mathrm{P}(\mathrm{x})$ is the prior probability of predictor.

\section{Steps in the algorithm:}

1. Conversion of dataset into frequency table.

2. Creation of likelihood table.

3. Use Naïve Bayesian equation to calculate posterior probability for each class, where the class with highest posterior probability is outcome of the prediction.

\section{CONCLUSION}

In this paper, we provide a survey and comparative study of existing techniques for opinion mining using machine learning and pattern based approaches. Research shows that machine learning methods such as naïve Bayes can give the highest accuracy and can be regarded as baseline learning method. We also studied the effect of various features of texts on classifier. We can concluded that whenever we use more cleaner data, we got more accurate results. We can focus on study of combining machine learning method into opinion mining method in order to improve the accuracy of sentiment classification and adaptive capacity to variety of domain and different language.

\section{REFERENCES}

[1]. W. Gao and F. Sebastiani, "Tweet sentiment: From classification to quantification," in Proc. IEEE/ACM Int. Conf. Adv. Social Netw. Anal. Mining (ASONAM), Aug. 2015, pp. 97-104.

[2]. J. M. Soler, F. Cuartero, and M. Roblizo, "Twitter as a tool for predicting elections results," in Proc. IEEE/ACM Int. Conf. Adv. Social Netw. Anal. Mining (ASONAM), Aug. 2012, pp. 1194-1200.

[3]. Jyoti Jain1, Prachi Panchal2, Nihar Suryawanshi3, Asst. Prof. Mrs. A.S. Shinde4,"Sentiment Analysis Using Supervised Machine Learning” 1,2,3,4, Department of Information Technology, Sinhgad Academy of Engineering, Kondhwa B.K., Pune -48

[4]. MONDHER BOUAZIZI AND TOMOAKI OHTSUKI, (Senior Member, IEEE,"A Pattern-Based Approach for Multi-Class Sentiment Analysis in Twitter" Graduate School of Science and Technology, Keio University, Yokohama 223-8522, Japan.

[5]. J. M. Soler, F. Cuartero, and M. Roblizo, "Twitter as a tool for predicting elections results," in Proc. IEEE/ACM Int. Conf. Adv. Social Netw. Anal. Mining (ASONAM), Aug. 2012, pp. 1194-1200.

[6]. Vishal A. Kharde Department of Computer Engg, Pune Institute of Computer Technology,Pune University of Pune (India) S.S. Sonawane Department of Computer Engg, Pune Institute of Computer Technology,Pune University of Pune (India) "Sentiment Analysis of Twitter Data: A Survey of Techniques" 